\title{
LOS SIMULADORES EDUCATIVOS COMO INSTRUMENTO PEDAGÓGICO PARA LA ENSEÑANZA DE LAS FINANZAS
}

\section{EDUCATIONAL SIMULATORS AS A PEDAGOGICAL TOOL FOR TEACHING FINANCE}

\author{
Mawency Vergel Ortega ${ }^{1}$ \\ Luisa Stella Paz Montes² \\ Diego Mauricio Álvarez $\mathrm{Paz}^{3}$ \\ Universidad El Bosque-UFPS
}

\section{RESUMEN}

El componente matemático es un aspecto relevante en el aprendizaje de las finanzas, ya que favorece el desarrollo de procesos cognitivos, tales como: razonamiento, visualización, interpretación, direccionamiento, relación, entre otros; que será útil para la comprensión de las diferentes situaciones; la complejidad de sus

$1 \quad$ Universidad Francisco de Paula Santander Cúcuta - Colombia, Orcid:0000-0001-8285-2968 Email: mawency@ufps.edu.co

2 Universidad Francisco de Paula Santander Cúcuta - Colombia, Orcid: 0000-0002-8887-3441 Email: luisastellapm@ufps.edu.co - Ipazmontes@gmail.com 3 Universidad El Bosque Bogotá - Colombia. Orcid.0000-0001-6731-8417, Email: dmalvarezp@unbosque. edu.co - diegoalvarezp17@gmail.com contenidos y la escasa aplicabilidad que los docentes promueven a partir de ellos, unido a la poca existencia de estrategias didácticas adecuadas que incentiven la motivación y el desarrollo de habilidades para comprenderlos, hacen que su estudio genere poca atracción por parte de los estudiantes.

La investigación buscó visualizar las competencias asociadas al pensamiento financiero mediante el uso de herramientas tecnológicas, en especial de simuladores, en los estudiantes de educación superior. Se enmarcó dentro del enfoque cualitativo, exploratorio, descriptivo y desarrollado bajo el método de 
Investigación Acción. Fueron seleccionadas como técnicas de recolección de información: observación directa, entrevista estructurada y encuesta. La población de investigación estuvo conformada por 25 estudiantes y 6 profesores que orientan las asignaturas correspondientes al área financiera. Entre los resultados se encontró que a través de la utilización de simuladores, los docentes pueden apropiarse de las TIC como mecanismo de enseñanza más asertivo para estimular el pensamiento financiero y económico en los estudiantes.

\section{PALABRAS CLAVE:}

simuladores, interpretación, datos, finanzas, contexto.

\section{ABSTRACT.}

The mathematical component is a relevant aspect in learning finance, since it favors the development of cognitive processes, such as: reasoning, visualization, interpretation, direction, relationship, among others; that will be useful for understanding the different situations; the complexity of its contents and the limited applicability that teachers promote from them, together with the limited existence of adequate didactic strategies that encourage motivation and the development of skills to understand them, mean that their study generates little attraction on the part of students.

The Project aims to visualize the skills associated with financial thinking through the use of technological tools, especially simulators, in higher education students. It was framed within the qualitative, exploratory, descriptive approach and developed under the Action Research method. They were selected as information gathering techniques: direct observation, structured interview and survey. The research population consisted of 25 students and 6 teachers who guide the subjects corresponding to the financial area. Among the results, it was found that through the use of simulators, teachers can appropriate ICT as a more assertive teaching mechanism to stimulate financial and economic thinking in students.

\section{KEYWORDS:}

simulators, interpretation, data, finance, context.

\section{INTRODUCCIÓN}

Las matemáticas y las finanzas han formado parte de la sociedad desde tiempos remotos, desde los ancestros siempre ha existido un esfuerzo por parte de la especie humana por mejorar su calidad de vida a través de una mejor distribución de sus ingresos y recursos. Los procesos asociados al pensamiento matemático constituyen un aspecto relevante en el aprendizaje del área financiera, puesto que favorecen el desarrollo de estructuras cognitivas en los estudiantes, como son: razonamiento, visualización, interpretación, entre otras. Su enseñanza no es tarea fácil, por la complejidad de sus contenidos y la escasa aplicabilidad que los docentes promueven de estos en contexto, más aún cuando no se manejan estrategias didácticas adecuadas que favorezcan la motivación y el desarrollo de habilidades en los discentes para la comprensión de estos contenidos.

En razón a esta situación y aprovechando la facilidad que los estudiantes tienen en el manejo de las nuevas tecnológicas de la información y la comunicación y conociendo los múltiples beneficios que éstas ofrecen a los procesos de enseñanza aprendizaje, se hace necesario optimizar su uso implementándolas como herramientas didácticas que favorezcan el desarrollo del pensamiento financiero. E proyecto tiene como propósito evidenciar el aporte significativo de los simuladores financieros, en el proceso de enseñanza y aprendizaje de dicha área y la necesidad de su utilización para lograr una mejor comprensión 
de los datos e indicadores aportados por las empresas o proyectos(Parra, Vergel, Suarez, 2019). En el diseño metodológico se desarrolló dentro de un enfoque cualitativo, puesto que se buscó observar la naturaleza de la realidad educativa para transformar las prácticas pedagógicas, con apoyo del enfoque cuantitativo apoyado en paradigma interpretativo para lo cual se tomó como punto de partida 6 docentes del área matemática y financiera y la utilización de herramientas tecnológicas para la enseñanza del pensamiento crítico, analítico e interpretativo y además se contó con la participación de 25 estudiantes de educación superior.

\section{MARCO TEÓRICO}

A partir de toda la revisión documental realizada para fundamentar la investigación, se encontró una serie de documentos, tesis, artículos, libros, entre otros estudios, que tienen una estrecha relación con los múltiples beneficios que proporciona el proceso de inclusión de los simuladores a la enseñanza-aprendizaje, especialmente en el desarrollo del pensamiento analítico y financiero en razón a la complejidad y aplicabilidad de sus contenidos. Adicional a esto, se presentan algunos estudios donde se resaltan las ventajas del uso de Tic como herramienta didáctica en distintas disciplinas del saber, teniendo en cuenta que son pocas las investigaciones que se han adelantado sobre la implementación de esta tecnología para favorecer específicamente el fortalecimiento de las competencias asociadas al pensamiento crítico e interpretativo.

Las investigaciones realizadas en la enseñanza de las matemáticas financieras mencionan que la dificultad para el aprendizaje de esta asignatura radica en la característica interpretativa e intrínseca que poseen. En los últimos 20 años, Colombia ha participado en algunas pruebas internacionales donde se ha evaluado el desempeño de los estudiantes en diferentes áreas. Una caracterización de estas pruebas, corroboran el bajo nivel de desempeño en las competencias analíticas e interpretativas, y entre estas las matemáticas y las financieras, situación que conlleva a las instituciones educativas a unir esfuerzos para mejorar el rendimiento de los colombianos en esta área. El análisis de dichos resultados reflejó que los estudiantes presentaban una gran dificultad para razonar y argumentar situaciones problema que requieren la aplicación del pensamiento matemático-financiero (López, Gómez, Vergel, 2019). Existe una estrecha relación entre errores, dificultades y obstáculos propuesta por Escamilla (2000) "dificultades se conectan y refuerzan en redes complejas que se concretan en la práctica en forma de obstáculos y se manifiestan en los alumnos en forma de errores".

Lo cual nos da una idea de jerarquía entre dichos conceptos para no seguir utilizándolos como sinónimos en los diferentes contextos y así poder diferenciarlos, caracterizarlos y comprenderlos de una manera más clara. Respecto a los obstáculos, es preciso citar a quien fuera el que propusiera la noción primera de Obstáculo, llamándolo Obstáculo Epistemológico, Gastón de Bachelard (1938) quien describe con claridad la importancia de tener en cuenta la experiencia y los conceptos adquiridos con anterioridad ante un conocimiento nuevo, asegurando que "cuando se presenta ante la cultura científica, el espíritu jamás es joven Hasta es muy viejo, pues tiene la edad de sus prejuicios". De esta forma se reconoce la resistencia que ponemos ante las ideas que contradicen nuestro conocimiento adquirido, tratando de poner por encima de ellas nuestros propios instintos, como si la opinión pesara ante la ciencia o si nuestras experiencias dieran cuanta absoluta de la realidad. Según Brousseau en contraposición a Bachelard (1986), los obstáculos se pueden caracterizar como conocimiento, el alumno utiliza este conocimiento para producir respuestas adaptadas en un cierto contexto que 
encuentra con frecuencia, cuando se usa este conocimiento fuera de este contexto genera respuestas incorrectas. Una respuesta universal exigiría un punto de vista diferente. El alumno resiste a las contradicciones que el obstáculo le produce y al establecimiento de un conocimiento mejor. Es indispensable identificarlo e incorporar su rechazo en el nuevo saber; después de haber notado su inexactitud, continúa manifestándolo, de forma esporádica.

Las TIC en educación. La Constitución Nacional de Colombia, en su artículo 20 reglamenta que toda persona se le garantiza la libertad de expresar y difundir su pensamiento y opiniones, la de informar y recibir información veraz e imparcial y la de fundar medios masivos de comunicación. En su artículo 67 establece la educación como un derecho de todas las personas y un servicio público que tiene función social; con ella se busca el acceso al conocimiento, a la ciencia, a la técnica y a los demás bienes y valores de la cultura. El Ministerio de las TIC. en la ley 1341, su artículo 39 reglamenta la articulación del Plan de TIC como apoyo al Ministerio de Educación Nacional para fomentar el emprendimiento en TIC, desde los establecimientos educativos, con alto contenido en innovación, poner en marcha un Sistema Nacional de alfabetización digital, capacitar en TIC a docentes de todos los niveles e incluir la cátedra de TIC en todo el sistema educativo.

Simuladores educativos. La integración de simuladores en la educación superior facilita afrontar más eficientemente el proceso de formación académica, fomentando un conocimiento analítico y práctico, asimismo ayuda a la adquisición de destrezas y habilidades en la incorporación del conocimiento tecnológico, apoyando los sistemas educativos.

Una de las principales funciones de los simuladores en educación es el apoyo a docentes en la transferencia de conocimiento. Bender y Fish (2000) mencionan una jerarquía de conocimiento cuando abordan la transferencia, y refieren los niveles siguientes: dato (mínima unidad de información), información (cuando se añade significado a los datos), conocimiento (cuando se da la aprehensión de hechos, verdades o principios), hasta la destreza (estadio superior cuando se trata de dar respuesta al porqué de las cosas y se generan habilidades y métodos de aplicación).

En el ámbito académico se han realizado diversos estudios para conocer la efectividad de los simuladores. Cabrera (2003), por ejemplo, realizó una investigación sobre el desarrollo de simuladores basados en casos y modelación dinámica para el sostenimiento de sistemas de calidad. Según el análisis de los resultados de los exámenes aplicados (Martínez, Vergel, Zafra, 2016), tanto al grupo de control como al de experimentación, se concluyó que existían marcadas diferencias en el aprendizaje entre los alumnos que usaron el simulador y los alumnos a quienes sólo se les aplicó el método del caso tradicional (Vergel, Gallardo, Martínez, 2014). El grupo con simulador mostró una mayor comprensión de la dinámica que daba origen a la problemática del caso, lo que se tradujo en respuestas más completas y precisas en el cuestionario de evaluación.

\section{METODOLOGÍA}

Para la realización del estudio, se optó por la aplicación del enfoque cualitativo, como lo respalda Hernández, Fernández, \& Baptista (2010), "un estudio cualitativo puede concebirse como un conjunto de prácticas interpretativas que hacen al mundo "visible", lo transforman y lo convierten en una serie de representaciones en forma de observaciones, anotaciones, grabaciones y documentos" (Wargner, 1982, p.23), y teniendo presente que en el enfoque cualitativo los individuos son agentes activos en la construcción de su realidad, serán los mismos docentes objeto de estudio los que la comprendan para mejorar su práctica de enseñanza del pensamiento matemático y financiero (Castilla, Jacome, Carrascal,2010). 
Se utilizó la observación directa, y la entrevista como técnica de recolección de datos para verificar, aprobar o rechazar relaciones entre las variables.

La investigación se basó en el método de investigación acción participante, como estrategia metodológica que involucra y sensibiliza a una comunidad con el conocimiento y la solución de problemas; cuya finalidad es la transformación social desde un interés emancipador. Se aplicó siguiendo los cuatro momentos o fases recomendadas por Latorre (2003): planificación, acción, observación y reflexión

Como estrategia de acción en el aula se utilizó la aplicación de encuestas para observar las estrategias didácticas 0 mediadas por las tecnologías y la utilización de simuladores que permitió integrar la teoría y la práctica, desarrollando en los estudiantes destreza para comprender procesos, establecer causas y escoger soluciones prácticas, ejercitar la actividad creadora, la iniciativa y criterio interpretativo. Bajo la consideración anterior, el diseño y aplicación de esta estrategia didáctica, posibilitó enriquecer la práctica pedagógica de los docentes del área financiera.

\section{RESULTADOS}

Analizadas todas las fuentes de recopilacion de informacion se obtiene que el $27,10 \%$ de los estudiantes afirma que el docente los orienta en la utilización de las herramientas tecnológicas, y un $23,28 \%$ que les enseña a utilizar los simuladores como estrategias para reforzar el aprendizaje, de lo cual se evidencia que los estudiantes reciben orientación por parte del docente, pero que estos confunden las herramientas tecnológicas con los simuladores gerenciales, además se observa un $17,81 \%$ que afirman no recibir orientaciones ni incentivar el uso de simuladores, y un $10,81 \%$ no utiliza las herramientas financieras ni los sofware financieros disponibibles toda vez que los docentes no incentivan o requieren su utilización.

Igualmente se encontró que el $44,18 \%$ de los docentes no utilizan en el desarrollo de sus asignaturas los simuladores financieros, toda vez que consideran que con la fundamentación teórica y los ejercicios realizados en clase son suficientes para afianzar los temas vistos. El $24,9 \%$ de los docentes entrevistados hizo referencia a lo didáctico de dicha estrategia, $19,22 \%$ argumento que en los ejercicios utilizan problemas y datos de la vida cotidiana de los estudiantes, datos de las empresas de la región y nacionales.

Con el proposito de finalizar la investigacion se propuso a los docentes utilizar los simuladores al final de la tematica de las asignauras a su cargo, propuesta que fue aceptada e implementada en el respectivo semestre. Con el desarrollo de la estrategia propuesta, los docentes afirmaron que pudieron constatar el grado de aceptación de dicha herramienta por parte de los estudiantes, además que permitió evidenciar la claridad en la comprensión de los conceptos y temas vistas, asi como la interpretacion de los resultados obtenidos y realizacion de diferentes pruebas de sensibilidad con los datos, con el objeto de obtener la situacion que le proporcionara mayor rentabilidad a la empresa y generara valor a la misma

Igualmente los estudiantes manifestaron sentirse muy a gusto con la implementación y utilización de dicha herramienta, toda vez que pudieron realizar cambios a los datos y visualizar los resultados que se obtienen al realizar modificaciones en los mismos o realizar diferentes inversiones, tal como sucede en la vida real en las empresas y en la parte personal.

Los estudiantes argumentaron que pese haber utilizado los simuladores en la parte final del programa académico, seria interesante y muy productivo utilizarlos en le trascurso del semestre, apreciacion que fue totalmete compartida por los docentes. En opinión 
generalizada tanto por parte de los estudiantes como de los docentes el uso de simuladores para mecanizar los conceptos y temas visto hace que las competencias se desarrollen y se alcancen con mayor rapidez y asertividad, ademas de lograr la participación de los alumnos en una forma realista y significativa.

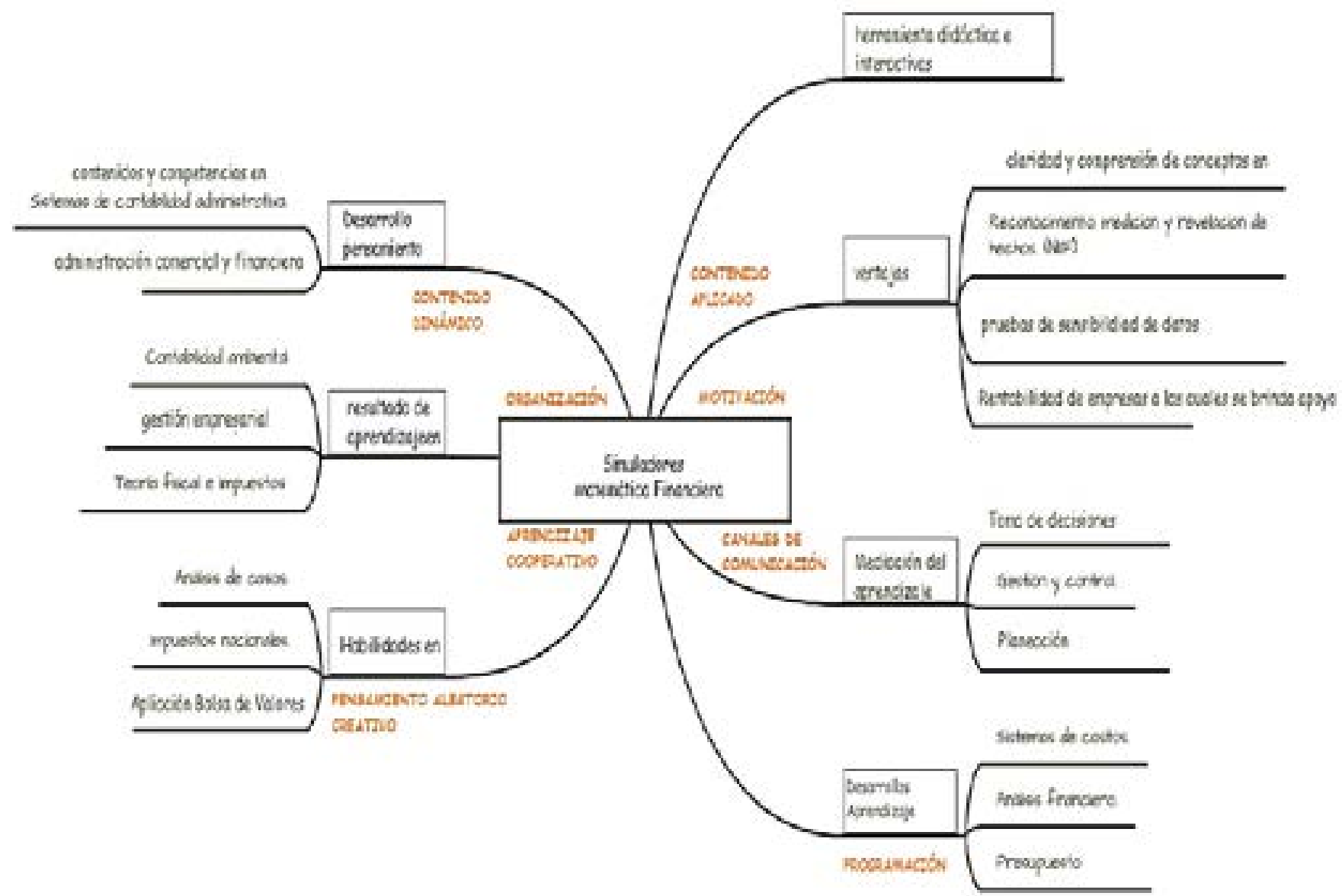

Figura 1. Categorías emergentes uso de simuladores. Fuente: Autores

Se constituyeron en categorias emergentes Herramienta didáctica, claridad conceptual, mediación, desarrollos, pensamiento, resultado de aprendizaje, habilidades asociados a familias contenido dinámico, aprendizaje cooperativo, pensamiento aleatorio, pensamiento crítico, canales de comunicación (Figura 1), motivación contenido aplicado. Subcategorías se asociaron a los temas vistos en matemática financiera y sus aplicaciones de acuerdo a talleres ejecutados $y$ visitas técnicas de auditoria y apoyo.

Se evidencia que el uso de los simuladores financieros y gerenciales son una herramienta mediadora del aprendizaje y que facilita la utilización de la información para generar situaciones deseables, es de fácil manejo y 
permite la ejercitación de algunos contenidos, adicionalmente se pueden generar canales de comunicación a través de comentarios para que el estudiante exprese sus puntos de vista, aclare dudas e inquietudes. Las plataformas web electrónicas se constituyen en una tecnología versátil, económica y fácil de utilizar. Presentan múltiples ventajas pedagógicas y formativas, dentro de las que este autor destaca: el encontrar, organizar y compartir información. Los simuladores se constituyen en herramienta útil para desarrollo de conceptos y construcción de conocimientos, así como para la aplicación de éstos a nuevos contextos, a los que el estudiante no puede acceder desde el contexto metodológico.

\section{CONCLUSIONES}

Se constituyeron en categorías emergentes del uso de simuladores en la enseñanza de la matemática financiera: la herramienta didáctica, claridad conceptual, mediación, desarrollos, pensamiento, resultado de aprendizaje, habilidades asociados a familias contenido dinámico, aprendizaje cooperativo, pensamiento aleatorio, pensamiento crítico, canales de comunicación

Para la enseñanza de las finanzas y específicamente de las matemáticas financieras la actitud del docente juega un papel determinante pues este debe centrar su atención en sus estudiantes, tener en cuenta las características e intereses del grupo, basarse en experiencias previas y aprovechar el contexto como fuente de aprendizaje.

El desarrollo de las tres competencias asociadas al pensamiento analítico y critico fueron comunicación asociado a canal y modelación, razonamiento y argumentación, planteamiento y resolución de problemas, se logró al relacionar cada una de las temáticas con la realidad y las situaciones del diario vivir, aprovechando al máximo los recursos del contexto y el discurso conceptual del docente apoyado en las herramientas tecnológicas, en especial los simuladores financieros, con el fin motivar y convertir al estudiante en artífice en la construcción de su propio conocimiento.

Aunque los docentes reconocen la importancia de las herramientas tecnológicas y de los simulares en las prácticas de enseñanza, en algunos se evidencia cierta apatía por incursionar en su utilización, ya sea por la exigencia que éstas demandan al seleccionar los recursos adecuados o por la responsabilidad de su manejo, desaprovechando las ventajas que pueden ofrecer para hacer del aula un laboratorio de construcciones propias y experiencias enriquecedoras que le permitirán al alumno familiarizarse con los diferentes situaciones problémicas que se le pueden presentar al interpretar los diferentes datos $\mathrm{e}$ indicadores financieros.

Por medio de la inclusión de las TIC, de los paquetes financieros $y$ en especial de los simuladores en los procesos formativos, el estudiante reconoce que no solo le sirven como herramientas de entretenimiento sino por el contrario son fuentes considerables de información, medio de comunicación y de ejercitación de sus saberes.

Los simuladores son una herramienta de gran potencial pedagógico en la medida en que es muy fácil de manejar, permite evaluar las diferentes situaciones que se pueden presentar y hacer los ajustes correspondientes de acuerdo a los resultados que se pretendan alcanzar.

Se puede decir, en forma general, que el uso de simuladores como estrategia didáctica, a través de los cuales se transfiere conocimiento, sí causa impacto en el proceso de aprendizaje de los estudiantes, puesto que las clases se vuelven más interesantes y llamativas, existiendo una mayor participación por parte de los alumnos, son más claras las explicaciones que se dan, incrementan la retención al presentarse los contenidos, y aumenta la motivación y el gusto por aprender y poner en práctica lo aprendido. 


\section{REFERENCIAS BIBLIORÁFICAS}

Bachelard Gastón (1983) Los obstáculos epistemológicos y meta cognitivos en matemáticas, Edit. Guzmán, Mx.

Bender, S. y Fish, A. (2000), "The transfer of knowledge and the retention of expertise: The continuing need for global assignments", Journal of Knowledge Management, 4 (2), pp. 125-137.

Cabrera, F. (2003), "Desarrollo de simuladores basados en casos y modelación dinámica para el sostenimiento de sistemas de calidad": Fecha de consulta: 17 de octubre de 2007. http://dinamica-sistemas.mty.itesm.mx/ congreso/ponencias pdf/26. simuladores. pdf

Castilla-Duarte, M. C. Jácome-Carrascal J. L. y Vergel-Ortega, M. "Emprendemat, su influencia en el desarrollo de competencias económicafinancieras en la juventud de la región del Catatumbo", Aibi revista de investigación, administración e ingeniería, vol. 8, no. 1, pp. 53-57, 2020.

Guzmán Tinajero, K., y Rojas-Drummond, S. M. Escritura colaborativa en alumnos de primaria: un modelo social de aprender juntos. Revista mexicana de investigación educativa, 17(52), 217-245. 2012

Escamilla, J. G. (2000), Selección y uso de tecnología educativa. México: Trillas.

G. Brosseau, «Fundamentos y métodos de la didáctica de la matemática,» Facultad de Matemática, Astronomía y Física. Univerisdad Nacional de Córdoba, Córdoba, 1986.

Hernández R Fernández C and Baptista P 2010 Metodología de la investigación Mc Graw Hill
Heering, P., \& Wittje, R. An Historical Perspective on Instruments and Experiments in Science Education. Science \& Education, 21(2), 151155. 2012.

Latorre, Antonio (2003), La investigación-acción: Conocer y cambiar la práctica educativa. España, GRAÓ

López Ovalle, E., Gomez Colmenares, C.A. y Vergel Ortega, M. Geocatatum: Its influence on the development of children's geometric thinking Journal of Physics: Conference series 1414, 2. 2019

Martínez J, Vergel M, Zafra S. 2016 Comportamiento juvenil y competencias prosociales Bogotá: Editorial Ibañez.

Parra, H., Suarez, J. and Vergel, M. 2019 Curricular trends in the University Francisco de Paula Santander academic program offerings, Journal of Physics: Conference Series 1329, 012013.

Peña, J.M. y Castellano, M.D. La lectura y la escritura en los estudiantes de instituciones educativas de Medellín participantes en el programa Prensa Escuela. Itinerario Educativo, 66, 225-246. 2015.

Pérez, J. J. (2005). Lectura y aprendizaje de las Matemáticas. Idea: revista de educación de Castilla-La Mancha, Toledo, año 1, n. 2, septiembre; p. 126-130

Pilonieta, L., Martínez, J. y Vergel, M. (2019). Lineamientos de enseñanza a estudiantes con hipoacusia: una acción creativa que transciende la educación inclusiva. Bogotá: Ecoe-Ediciones.

Rojas Y. (2019). Multiplicación en primera infancia. Revista covalente. 1 (1). 42-46

Rodríguez Cancio, M. (2011). Materiales y recursos en Educación Infantil. Manual de usos prácticos para el docente. Vigo. Ed: Ideas Propias. 
Santiago, M., Vergel-Ortega, M. y Rojas J.P. (2019). Mathematics, resilience and development of thinking of youth. Journal of Physics Conference Series 1408 (1) 012012

Torres-Ortiz, J. Incidencia de la Moodle en las prácticas en modalidad educativa B-Learning. Investigación, Desarrollo e Innovación, 3948. 2012

Vergel, M., Gallardo, H. \& Martínez, J. (2014). Factores asociados al rendimiento académico en estadística de estudiantes de administración pública. Bogotá: Colección Pedagogía Iberoamericana.

Vergel Ortega, M., Martínez Lozano, J., \& Zafra Tristancho, S. (2016). Cultivo de cebolla y su comportamiento en la provincia de ocaña. Revista Colombiana de Ciencias Hortícolas, 10(2), 333-344.

Wagner, S. J. (1982). A longitudinal analysis of early number concepts: From numbers to number. Nueva York: Action and Thought 